\title{
ESTIMATIVA DE TEMPERATURAS BASAIS E EXIGÊNCIA TÉRMICA EM MANGUEIRAS NO NORDESTE DO ESTADO DO PARÁ ${ }^{1}$
}

\author{
JULIANA CHAGAS RODRIGUES², PAULO JORGE DE OLIVEIRA PONTE DE SOUZA ${ }^{3}$, \\ RENATA TRINDADE DE LIMA ${ }^{4}$
}

RESUMO - O objetivo deste trabalho foi estimar as temperaturas basais e a exigência térmica da mangueira, na região nordeste do Estado do Pará. Um experimento foi realizado em um pomar de mangueiras de 25 hectares, durante as safras de 2010/2011 e 2011/2012, sendo acompanhadas diariamente a fenologia e as condições meteorológicas desde a floração até a colheita. As duas safras experimentaram diferentes condições meteorológicas, e, por isso, a duração do ciclo e a exigência térmica diferiram significativamente. As temperaturas basal inferior e superior para a cultura da mangueira foram de $13{ }^{\circ} \mathrm{C} \mathrm{e} 32^{\circ} \mathrm{C}$, respectivamente, a exigência térmica média encontrada para a mangueira atingir o ponto de colheita comercial foi de 1.024,23 ${ }^{\circ} \mathrm{C}$ dia $^{-1}$ a $1.157,99{ }^{\circ} \mathrm{C}$ dia $^{-1}$ e a duração média do ciclo da mangueira foi de 110 a 112 dias.

Termos para indexação: Mangifera indica, Tommy Atkins, fenologia, graus-dia.

\section{ESTIMATE OF BASE TEMPERATURE AND THERMAL REQUIREMENT IN MANGO FOR THE NORTHEAST OF PARÁ}

\begin{abstract}
The objective of this study was to estimate the threshold temperatures and the thermal requirement of mango, in the northeast of the state of Para. One experiment was carried out in a mango orchard of 25 hectare during the 2010/2011 and 2011/2012 season in which the phenological and meteorological conditions were daily monitored from flowering to harvest. Both mango seasons were submitted to different meteorological conditions, and therefore, both the duration of the reproductive stage and the mean thermal requirement were significantly different. The thresholds temperatures (lower and higher) of the mango were $13{ }^{\circ} \mathrm{C}$ and $32{ }^{\circ} \mathrm{C}$, respectively, the thermal requirement found for the mango orchard reach to the harvest stage was $1,024.23{ }^{\circ} \mathrm{C}$ day ${ }^{-1}$ and $1,157.99^{\circ} \mathrm{C}$ day $^{-1}$, and the mean duration of the mango season was 110 and 112 days.
\end{abstract}

Index terms: Mangier indicia, Tommy Atkins, phenology, degree-days.

\section{INTRODUÇÃO}

A temperatura do ar é uma das variáveis ambientais que mais interferem no desenvolvimento das plantas, especialmente em casos em que não ocorra deficiência hídrica acentuada, tendo efeito direto sobre o metabolismo das plantas (RIBEIRO et al., 2006; LUCAS et al., 2012).

A mangueira tolera temperaturas entre $-6^{\circ} \mathrm{C}$ e $40^{\circ} \mathrm{C}$, existindo variedades que suportam até 48 ${ }^{\circ} \mathrm{C}$. Fora deste intervalo, ocorrem danos em plantas adultas e morte de plantas jovens (CAMPBELL; CAMPBELL, 2006; RAMÍREZ; DAVENPORT, 2010). O florescimento da mangueira, em condições subtropicais e tropicais de altitude, depende da ocorrência de temperaturas frias, sendo que estas devem ocorrer durante todo o processo de florescimento, desde a morfogênese floral até a formação completa da inflorescência. $\mathrm{O}$ efeito de baixas temperaturas nestas regiões é evidente, diferindo das regiões de baixa latitude, onde o florescimento é menos dependente da temperatura, sendo necessária a exposição da planta a um moderado estresse hídrico (RAMÍREZ; DAVENPORT, 2010).

Deste modo, estudos mostram que temperaturas mais altas, entre $25^{\circ} \mathrm{C}$ e $30^{\circ} \mathrm{C}$, proporcionam maior desenvolvimento vegetativo da planta, enquanto temperaturas mais amenas, entre $10{ }^{\circ} \mathrm{C}$ e $18{ }^{\circ} \mathrm{C}$, promovem o desenvolvimento de gemas reprodutivas (NUÑEZ-ELISEA et al., 1996), havendo ótimo crescimento da mangueira entre $24^{\circ} \mathrm{Ce} 30^{\circ} \mathrm{C}$, pois tanto as temperaturas muito baixas quanto as muito elevadas prejudicam o crescimento, o desenvolvimento e a produção, além de afetar a qualidade dos frutos.

'(Trabalho 169-12). Recebido em: 24-05-2012. Aceito para publicação em: 29-11-2012.

${ }^{2}$ Eng. Agrônoma, MSc. Agronomia, UFRA, Belém-PA - E-mail: juliana.rodrigues@ufra.edu.br

${ }^{3}$ Meteorologista, Dr. Prof. Adjunto, Instituto Sócio Ambiental e dos Recursos Hídricos - ISARH, UFRA, Belém-PA - E-mail: paulo. jorge@ufra.edu.br.

${ }^{4}$ Eng. Agrônoma, MSc. Agronomia, UFRA, Belém-PA - E-mail: renata.lima@ufra.edu.br. 
Uma metodologia bastante utilizada para se estimar o tempo necessário entre o florescimento e a maturação do fruto são as unidades térmicas (grausdia) ou exigência térmica da cultura, que representam o acúmulo diário de energia, acima da condição mínima e abaixo da máxima exigida pela planta. Este método pode auxiliar no planejamento de algumas atividades agrícolas, como o plantio, a colheita e os demais tratos culturais, e indicar o potencial climático de uma região para produção (SOUZA et al., 2009).

Para utilização deste método, torna-se necessário o conhecimento das temperaturas basal inferior e superior da cultivar, temperaturas abaixo e acima, respectivamente, da qual seu crescimento é desprezível ou nulo, sendo estes os limites para o desenvolvimento de vegetais (SOUZA et al., 2009).

Vários métodos estatísticos, como o menor desvio-padrão em graus-dia e em dias, o coeficiente de variação e o da regressão (YANG et al., 1995) são utilizados para a determinação das temperaturas basais inferior e superior de uma cultura. Tais métodos são os mais utilizados apesar de sofrerem críticas por considerar o desenvolvimento do vegetal linear, desprezando os fatores bióticos e abióticos que podem afetá-los e limitar seu desenvolvimento (STRECK, 2002).

Vários autores estudaram o uso dos grausdia acumulados para determinar o desenvolvimento de culturas, como mamão (BERILLI et al., 2007), abacaxi (CARVALHO et al., 2005), uva (CHAVARRIA et al., 2009), figo (SOUZA et al., 2009), manga (BARROS et al., 2010) e melancia (TRENTIN et al., 2008), não havendo, no entanto, trabalhos para condições de cultivo na Amazônia. Considerando os aspectos apresentados, este trabalho teve como objetivo estimar as temperaturas basais e a exigência térmica da mangueira em função da fenologia, na região nordeste do Estado do Pará.

\section{MATERIAL E MÉTODOS}

O experimento foi conduzido em um pomar de mangueiras localizado no sítio experimental de Cuiarana, no município de Salinópolis, Pará $\left(00^{\circ} 39^{\prime} 50,50{ }^{\prime} \mathrm{S}, 47^{\circ} 17^{\prime} 4,10^{\prime} \mathrm{O}\right)$. De acordo com a classificação de Köppen, o tipo climático predominante na região é Aw, com reduzida amplitude térmica, e pluviosidade anual de 2.100 $\mathrm{mm}$, sendo que $90 \%$ dessa pluviosidade se distribuem nos seis primeiros meses do ano.

O pomar foi implantado para fins comerciais, em 1993, e é constituído da variedade Tommy Atkins, utilizando o espaçamento de 10,0 x 10,0 m,

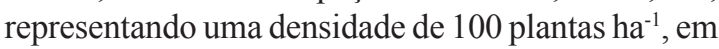

uma área total de 25 ha, sem irrigação.

Uma área correspondente a um hectare foi demarcada para o estudo, no centro da qual foi instalada e instrumentada uma torre micrometeorológica com $10 \mathrm{~m}$ de altura, apresentando um "fetch" superior à razão 1:100, para evitar que os efeitos advectivos interfiram nas medidas realizadas no pomar (RANA; KARTERJI, 2000).

As temperaturas foram medidas por Termoigrômetros (HMP45, Vaisala) instalados a dois metros acima do dossel, estando conectados a um datalogger (CR10X, Campbell Scientific) com leituras feitas a cada 10 segundos, e gravação das médias, a cada 10 minutos. O período utilizado para as medições compreendeu os meses de setembro de 2010 a fevereiro de 2011, e de julho de 2011 a janeiro de 2012.

A coleta de dados foi realizada durante as safras de 2010/2011 e 2011/2012, abrangendo os estádios fenológicos do ciclo produtivo da mangueira, os quais, segundo Soares et al. (1998), são compostos por quatro fases, representadas pela floração, queda, formação e maturação de frutos (Tabela 1). Para o acompanhamento da fenologia, foram selecionadas aleatoriamente 25 plantas, representando $25 \%$ do hectare previamente demarcado. A determinação das fases fenológicas foi estabelecida quando $50 \%$ +1 do total de plantas selecionadas apresentou o estádio observado.

A temperatura basal inferior ( $\mathrm{Tb}$ ) foi determinada de acordo com o método 1 descrito por Tretin et al. (2008) (Equação 1), sendo obtida em função das necessidades térmicas, para cada fase fenológica, e o ciclo reprodutivo total da mangueira, para cada planta em estudo, utilizando temperaturas do ar preestabelecidas entre $6{ }^{\circ} \mathrm{C}$ e 20 ${ }^{\circ} \mathrm{C}$, em intervalos de $1{ }^{\circ} \mathrm{C}$, em $1{ }^{\circ} \mathrm{C}$ como sendo a $\mathrm{Tb}$. Em função das necessidades térmicas obtidas, foram determinados os coeficientes de variação (CV) referentes a cada temperatura utilizada, sendo considerada a $\mathrm{Tb}$ aquela que correspondeu ao menor CV, conforme Barros et al. (2010).

$$
G D=\sum\left[\frac{(T \max -T \min )}{2}\right]-T b
$$

em que GD: são os graus-dia acumulados $\left({ }^{\circ} \mathrm{C}\right.$ dia $\left.^{-1}\right)$; Tmáx é a temperatura máxima do ar $\left({ }^{\circ} \mathrm{C}\right)$; Tmín é a temperatura mínima do ar $\left({ }^{\circ} \mathrm{C}\right)$ e Tb é a temperatura basal inferior $\left({ }^{\circ} \mathrm{C}\right)$.

Após a obtenção da $\mathrm{Tb}$, a temperatura basal superior (TB) foi estabelecida utilizando-se dos casos 2 (Equação 2) e 4 (Equação 3), descritos por Prela 
e Ribeiro (2002), pois foram os que abrangeram as condições térmicas no período do experimento. Para a obtenção da TB, utilizou-se o mesmo período que a $\mathrm{Tb}$, para cada planta em estudo, empregando temperaturas do ar predeterminadas entre $25^{\circ} \mathrm{C}$ e 35 ${ }^{\circ} \mathrm{C}$, em uma variação de $1{ }^{\circ} \mathrm{C}$, em $1{ }^{\circ} \mathrm{C}$, sendo que a TB foi encontrada quando o CV se tornou constante, de acordo com Souza et al. (2009).

A exigência térmica do ciclo reprodutivo da mangueira, para as duas safras, foi então calculada pela metodologia usada por Prela e Ribeiro (2002), cujos casos são descritos abaixo:

$\mathbf{1}^{\circ}$ Caso: Tb $<$ Tmín; Tmáx $<$ TB

$$
G D=\left(\frac{T \max -T \min }{2}\right)+(T \min -T b)
$$

$$
\begin{gathered}
4^{\circ} \text { Caso: } \mathrm{Tb}<\mathrm{Tmín} ; \mathrm{TB}<\text { Tmáx } \\
G D=\frac{2 *(T \max -T \min ) *(T \min -T b)+(T \max -T \min )^{2}-(T \max -T B)^{2}}{2 *(T \max -T \min )}
\end{gathered}
$$

em que: TB é a temperatura basal superior

As variáveis de duração e acúmulo de grausdia dos subperíodos fenológicos foram submetidas à análise de variância, e as médias, comparadas pelo teste de Tukey, a 5\% de significância, além da utilização de estatística descritiva através do uso de médias, desvio e erro-padrão.

\section{RESULTADOS E DISCUSSÃO}

O início do florescimento nas safras de 2010/2011 e 2011/2012 aconteceu em 10 de outubro e 20 de setembro, respectivamente, sendo finalizados em 27 de janeiro, para a primeira safra, e 9 de janeiro para a segunda safra, e as condições meteorológicas durante o experimento são apresentadas na Tabela 2. A temperatura média do ar para todo o período reprodutivo foi de $27,4^{\circ} \mathrm{C}( \pm 1,28)$ e $27,6^{\circ} \mathrm{C}( \pm 0,47)$, na primeira e na segunda safras, respectivamente, apresentando total de chuvas de 434,34 $\mathrm{mm}$, na primeira safra, e de $141,99 \mathrm{~mm}$, na segunda safra.

A safra de 2010/2011 foi exposta à condição de temperaturas mais elevadas, proporcionando o acúmulo mais rápido de graus-dia, tendo como consequência menor duração do que a segunda safra. Devido à ocorrência de menor amplitude térmica na segunda safra, a mangueira necessitou de mais tempo para acumular a exigência térmica necessária para completar seu ciclo produtivo, condição semelhante foi observada por Souza et al. (2009) para a cultura do figo e por Barros et al. (2010) para da mangueira.
Durante o período anterior ao início da fase reprodutiva da mangueira, é importante a observação das condições meteorológicas da região de cultivo, uma vez que estas influenciam diretamente na produtividade do pomar. Assim, em condições tropicais, o florescimento da mangueira ocorre após um período de plena vegetação, sendo este influenciado por um momento de moderado estresse hídrico (BALLY et al., 2000; RAMÍREZ; DAVENPORT, 2010).

As safras de 2010/2011 e de 2011/2012 foram expostas a condições meteorológicas bem diferenciadas (Figura 1). A primeira safra foi antecedida por uma brusca redução na precipitação pluviométrica da região, com $26 \mathrm{~mm}$ em agosto e $4 \mathrm{~mm}$ em setembro. Consequentemente, as temperaturas médias do ar foram bem elevadas nestes meses, variando entre $28,1{ }^{\circ} \mathrm{C}$ e $28,4{ }^{\circ} \mathrm{C}$. Ao longo da safra de 2010/2011, as temperaturas absolutas do ar variaram de $20,6{ }^{\circ} \mathrm{C}$ a $31,6{ }^{\circ} \mathrm{C}$, com máxima amplitude térmica chegando a 10,0 ${ }^{\circ} \mathrm{C}$, e amplitude térmica média de $5,2{ }^{\circ} \mathrm{C}$. As fases de formação e maturação apresentaram as maiores amplitudes térmicas com médias de $5,8^{\circ} \mathrm{C}$ e $5,7^{\circ} \mathrm{C}$, respectivamente, representando maior variabilidade na temperatura neste período, fato este causado pela concentração das chuvas a qual apresentou o total de $434,34 \mathrm{~mm}$.

Na safra de 2010/2011, cerca de 324,10 $\mathrm{mm}(74,62 \%$ do total no ciclo) ocorreram de forma concentrada durante a fase de maturação dos frutos, ocasião em que esta necessita de uma redução nas chuvas, uma vez que é a fase onde ocorre o acúmulo de açúcares, tornando o fruto mais adocicado. Esta concentração nas chuvas promoveu a ocorrência de frutos maiores, com peso médio de $581 \mathrm{~g}$, e redução na produtividade e número médio de frutos por planta, que foi de 11,85 tha ${ }^{-1}$ e 212 frutos, respectivamente, sendo ambos os parâmetros comparados com a safra de 2011/2012.

A segunda safra foi antecedida por uma redução brusca na precipitação pluviométrica da região, julho $(233 \mathrm{~mm})$ e agosto $(34 \mathrm{~mm})$; em decorrência disso, as temperaturas médias também foram mais elevadas, variando entre $26,2{ }^{\circ} \mathrm{C}$ e $27,1^{\circ} \mathrm{C}$. Este cenário resultou no estresse hídrico necessárioà cultura para iniciar seu florescimento, que foi antecipado em relação à safra anterior (Tabela 1). Durante essa safra, as temperaturas absolutas variaram entre $20,2^{\circ} \mathrm{C}$ e $30,8^{\circ} \mathrm{C}$, com a amplitude térmica máxima de $10,6{ }^{\circ} \mathrm{C}$, e média de $4,3{ }^{\circ} \mathrm{C}$, sendo a fase de queda de frutos a que teve a maior amplitude térmica média $\left(4,8^{\circ} \mathrm{C}\right)$. As demais fases apresentaram oscilação na amplitude térmica em 
torno de $4,0^{\circ} \mathrm{C}$; no entanto, ela foi menor que na safra anterior.

A temperatura média registrada ao longo do ciclo reprodutivo de $2011 / 2012$ foi de $27,6^{\circ} \mathrm{C}$ $( \pm 0,47)$, com baixo desvio-padrão, resultado da pequena variabilidade. Isto se deveu à escassez das chuvas, que foi de apenas 141,99 mm, valor bem inferior ao ocorrido na safra de 2010/2011. Neste ano, $74 \%$ do total $(104,90 \mathrm{~mm})$ estiveram concentradas na fase de queda de frutos com contínua redução no total de chuvas, nas fases fenológicas subsequentes, o que pode ter contribuído para maior produtividade média $\left(19,46 \mathrm{t} \mathrm{ha}^{-1}\right)$, com número médio de frutos por planta igual a 375 , mas apresentando frutos menores, com peso médio dos frutos de $518 \mathrm{~g}$.

Foram obtidos valores de $\mathrm{Tb}$ para cada fase fenológica, sendo que apenas as fases de quedaformação e maturação-colheita apresentaram resultados consistentes com a literatura consultada, apresentando $18,0{ }^{\circ} \mathrm{C}$ e $13,0{ }^{\circ} \mathrm{C}$, respectivamente. $\mathrm{O}$ valor de $\mathrm{Tb}$ encontrado para o intervalo quedaformação, correspondente a $18,0{ }^{\circ} \mathrm{C}$, sendo considerado elevado quando comparado com outros autores em estudos realizados no Brasil, mas ficou próximo daquele encontrado na Palestina, por Oppenheimer (1947), citado por Burondkar et al. (2000), que foi de $17,9^{\circ} \mathrm{C}$.

Um dos motivos causadores da dificuldade na obtenção da temperatura basal pode estar ligado à pouca variabilidade na temperatura, em algumas subfases, pois a reduzida amplitude térmica leva à obtenção de valores de coeficientes de variação muito próximos, originando valores absurdos, totalmente incoerentes com aqueles apresentados pela literatura, além da impossibilidade de gerar a curva característica da temperatura basal. As fases de queda-formação e maturação-colheita ocorreram em condições meteorológicas bem distintas, em relação às demais subfases, uma vez que os dados obtidos neste período se encontravam na transição do período seco (queda-formação) para o chuvoso (maturação-colheita).

Por conta disto, a $\mathrm{Tb}$ encontrada para o intervalo maturação-colheita, $13,0^{\circ} \mathrm{C}$, foi considerada mais coerente, sendo, portanto, utilizada nos cálculos (Figura 2), além de ter sido adotado um valor único para todo o ciclo da cultura por tornar sua aplicação mais fácil (CAMARGO et al., 1987; ANDRADE et al., 2005). Este valor ficou próximo ao encontrado por Mosqueda-Vásquez et al. (1993), no México, $12,0^{\circ} \mathrm{C}$, e Barros et al. (2010), na região da Baixada Cuiabana, no Brasil, $10^{\circ} \mathrm{C}$, para as variedades Manila e Alfa, respectivamente. O uso de diferentes métodos, associado a dados inconsistentes de fenologia, levam a diferentes estimativas da temperatura-base, devido à mesma ser definida através de critérios estatísticos ao invés de fisiológicos (YANG et al., 1995).

Os trabalhos de estimativa de temperaturas basais para diversas culturas, normalmente, calculam apenas a $\mathrm{Tb}$, pois são conduzidos em regiões onde raramente se atinge a TB, fato inverso é comumente observado na região tropical.

Baseado na metodologia proposta, a TB encontrada para a fase queda-formação foi de $32^{\circ} \mathrm{C}$ (Figura 3). Devido à não consistência dos resultados obtidos para as demais fases fenológicas, este valor foi utilizado em todos os cálculos, estando muito próximo do encontrado por Issarakraisila e Considine (1994) para a variedade Kensington, na Austrália, 33 ${ }^{\circ} \mathrm{C}$. Segundo os autores, temperaturas acima de $33^{\circ} \mathrm{C}$ prejudicam a formação do grão de pólen, reduzindo em $50 \%$ sua viabilidade.

A exigência térmica encontrada para a manga, desde a antese até o ponto de colheita comercial, variou entre $1.024,23{ }^{\circ} \mathrm{C} \mathrm{dia}{ }^{-1}$ e $1.157,99{ }^{\circ} \mathrm{C}$ dia $^{-1}$ (Tabela 3). Estes valores apresentaram diferenças significativas $(\mathrm{p}<0,05)$ e foram menores que os de outros trabalhos realizados para a cultura da manga em outras regiões do Brasil. Barros et al. (2010), baseados na mesma metodologia empregada neste trabalho, no entanto, abrangendo o período desde o início da frutificação até a colheita, encontraram, 2.116,50 ${ }^{\circ} \mathrm{C} \mathrm{dia}{ }^{-1}$ para uma $\mathrm{Tb}$ de $10^{\circ} \mathrm{C}$. Também, no Brasil, Lucena et al. (2007) utilizaram a metodologia para o acúmulo de graus-dia, baseada na diferença entre a temperatura média do ar e a Tb, com Tb igual a $10{ }^{\circ} \mathrm{C}$, e encontraram para a variedade Tommy Atkins em Petrolina, Pernambuco, uma exigência térmica de $1.939,70{ }^{\circ} \mathrm{C}$ dia $^{-1}$ para o período, desde a antese até a maturidade fisiológica.

Os valores apresentados na literatura são variados, mostrando a diversidade das condições edafoclimáticas das regiões estudadas, temperatura base utilizadas, manejo da cultura e a utilização ou não da irrigação nos cultivos. Além disso, não foram encontrados trabalhos que determinassem a exigência térmica para as fases fenológicas da mangueira, apenas para o ciclo total. Ao comparar os valores da exigência térmica encontrada neste trabalho com outros experimentos realizados no Brasil, foram observadas diferenças significativas entre eles, sendo que esta variabilidade pode ter ocorrido devido às distintas condições meteorológicas a que foram expostas em cada caso.

As durações do ciclo reprodutivo da mangueira apresentaram diferença significativa $(\mathrm{p}<0,05)$, variando entre 110 e 112 dias. Os valores referentes às fases fenológicas são apresentados 
na Tabela 3. Os resultados encontrados foram semelhantes ao obtido por Barros et al. (2010), que observaram 112 dias, mas diferiram dos resultados de Lucena et al. (2007), que consideraram a maturidade fisiológica da manga sendo atingida aos 98 dias após a antese. A duração média do ciclo reprodutivo esteve dentro do intervalo encontrado por Burondkar et al. (2000), que variou entre 93 e 127 dias para as cultivares Alphonso, Kesar e Ratna.

TABELA 1 - Fases fenológicas da mangueira.

\begin{tabular}{ll}
\hline \multicolumn{1}{c}{ Fase fenológica } & \multicolumn{1}{c}{ Descrição } \\
\hline Floração & $\begin{array}{l}\text { Panículas totalmente abertas. } \\
\text { Queda }\end{array}$ \\
$\begin{array}{l}\text { Frutos na planta e caídos no chão em tamanho de "cabeça de alfinete", "bola de gude" } \\
\text { e "ovo ou bola de bilhar". }\end{array}$ \\
$\begin{array}{l}\text { Frutos na planta em tamanho maior que "ovo ou bola de bilhar" e coloração da casca } \\
\text { Maturação }\end{array}$ & $\begin{array}{l}\text { Frutos na planta em tamanho "fruto" e coloração da casca esverdeada para verde- } \\
\text { arroxeada. }\end{array}$ \\
\hline
\end{tabular}

TABELA 2 - Início das fases fenológicas da mangueira, temperaturas absolutas máximas e mínimas, e médias, em ${ }^{\circ} \mathrm{C}$, e totais de chuva, em mm, durante o experimento.

\begin{tabular}{|c|c|c|c|c|c|c|c|c|c|c|}
\hline \multirow{2}{*}{ Fase fenológica } & \multicolumn{5}{|c|}{ Safra 2010/2011 } & \multicolumn{5}{|c|}{ Safra 2011/2012 } \\
\hline & Início & Tmáx & Tmín & $\mathbf{T}$ & Chuva & Início & Tmáx & Tmín & $\mathbf{T}$ & Chuva \\
\hline Floração & $10-10$ & 31,50 & 23,30 & 28,12 & 1,27 & 20-09 & 30,82 & 25,13 & 27,59 & 0,00 \\
\hline Queda & $05-11$ & 31,62 & 23,00 & 28,32 & 19,30 & $16-10$ & 30,60 & 20,17 & 27,39 & 104,90 \\
\hline Formação & $07-12$ & 31,57 & 22,43 & 27,33 & 89,66 & $18-11$ & 30,62 & 24,23 & 27,60 & 15,24 \\
\hline Maturação & 03-01 & 30,47 & 20,63 & 25,73 & 324,10 & $15-12$ & 30,77 & 20,87 & 27,66 & 21,84 \\
\hline
\end{tabular}

TABELA 3 - Duração e exigência térmica por fase (GD por fase) e acumulada (GD acumulado) da mangueira cv. Tommy Atkins durante os dois anos do experimento.

\begin{tabular}{|c|c|c|c|}
\hline \multirow{2}{*}{ Fase fenológica } & Duração & GD por fase & GD acumulado \\
\hline & --- dias ----- & \multicolumn{2}{|c|}{---- ${ }^{\circ} \mathrm{C} \mathrm{dia}^{-1}$---- } \\
\hline Floração & $26 a^{*}$ & $267,05 \mathrm{a}^{*}$ & $10,08( \pm 0,23)$ \\
\hline Queda & $32 \mathrm{~b}$ a $33 \mathrm{a}$ & $332,08 \mathrm{a}$ & $276,73( \pm 3,85)$ \\
\hline Formação & $27 \mathrm{a}$ & $227,80 \mathrm{~b}$ a $285,82 \mathrm{a}$ & $608,47( \pm 5,45)$ \\
\hline Maturação & $25 \mathrm{~b}$ a $26 \mathrm{a}$ & $194,82 \mathrm{~b}$ a $279,10 \mathrm{a}$ & $862,21( \pm 7,57)$ \\
\hline Total & $110 \mathrm{~b}$ a $112 \mathrm{a}$ & 1024,23 b a $1157,99 a$ & $1091,11( \pm 8,27)$ \\
\hline
\end{tabular}

*Médias seguidas pela mesma letra na linha, para duração e GD por fase, não diferem entre si, pelo teste de Tukey, a 5\% de significância. Os valores entre parênteses representam o erro-padrão. 


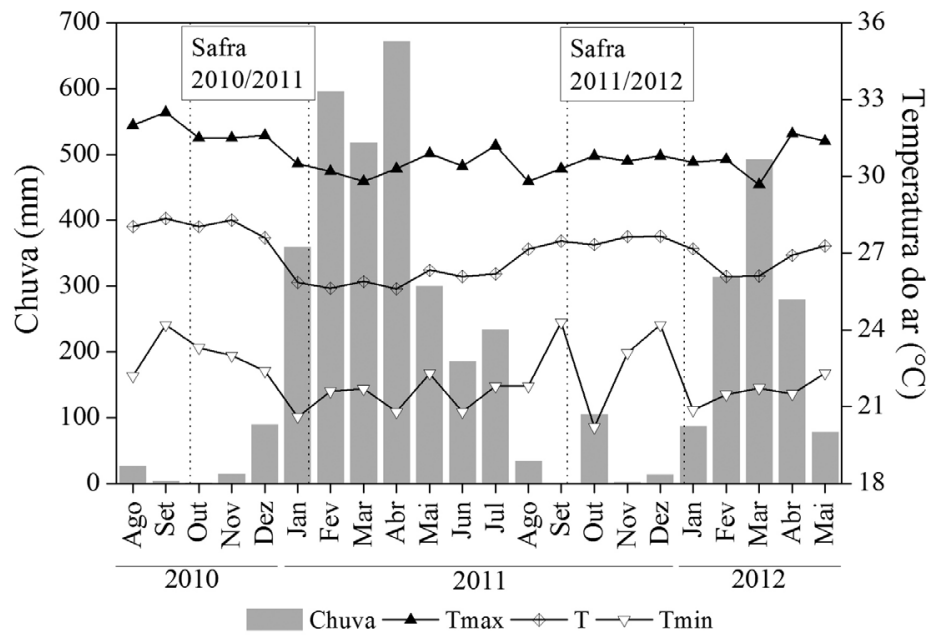

FIGURA 1 - Variabilidade das chuvas e das temperaturas máximas (Tmáx), médias (T) e mínimas (Tmín), durante o experimento, entre agosto de 2010 e maio de 2012.

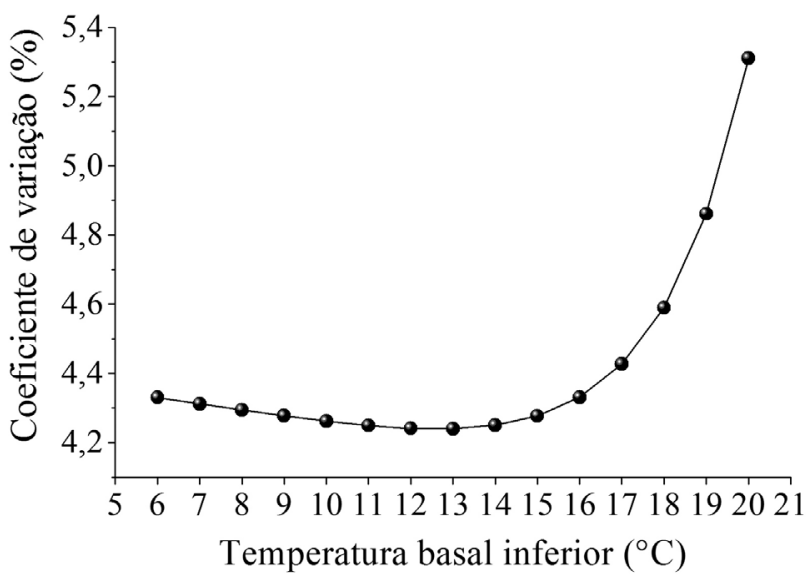

FIGURA 2 - Determinação da temperatura basal inferior pelo método do coeficiente de variação dos grausdia, para mangueira cv. Tommy Atkins.

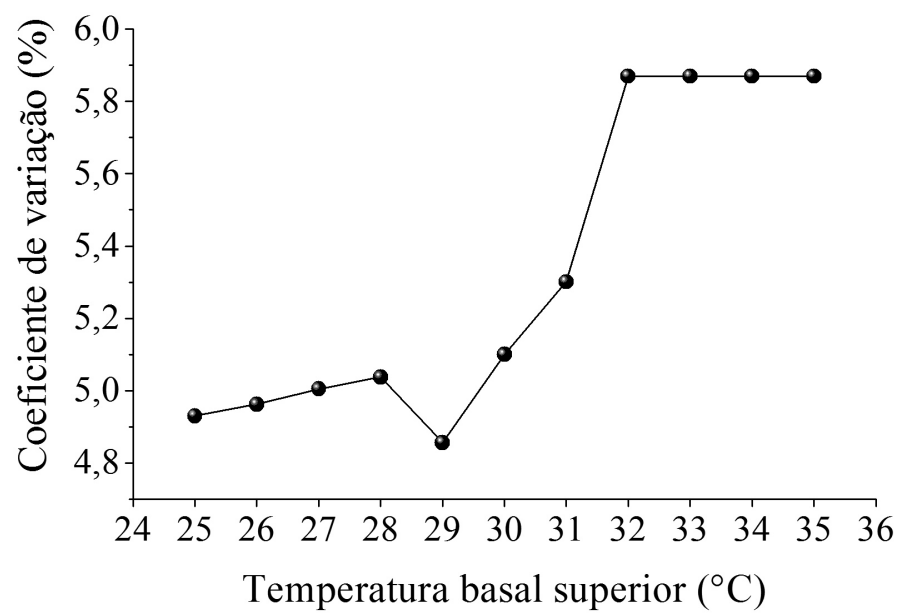

FIGURA 3 - Determinação da temperatura basal superior pelo método do coeficiente de variação dos grausdia, para mangueira cv. Tommy Atkins. 


\section{CONCLUSÕES}

1- As temperaturas basal inferior e superior encontradas pelo método do coeficiente de variação foram de $13{ }^{\circ} \mathrm{C}$ e $32{ }^{\circ} \mathrm{C}$, respectivamente.

2-A exigência térmica estabelecida para mangueira variou entre $1.024,23{ }^{\circ} \mathrm{C} \mathrm{dia}^{-1} \mathrm{e} 1.157,99$ ${ }^{\circ} \mathrm{C} \mathrm{dia}^{-1}$, para o ciclo produtivo, sendo que as médias necessárias para o início de cada fase fenológica são $10,08^{\circ} \mathrm{C} \mathrm{dia}{ }^{-1}, 276,73{ }^{\circ} \mathrm{C} \mathrm{dia}{ }^{-1}, 608,47{ }^{\circ} \mathrm{C}$ dia $^{-1}$ e $862,21{ }^{\circ} \mathrm{C}$ dia $^{-1}$ para floração, queda, formação e maturação dos frutos, respectivamente. A exigência térmica média acumulada em cada fase foi de 267,05 ${ }^{\circ} \mathrm{C}$ dia $^{-1}$, na floração; $332,08{ }^{\circ} \mathrm{C} \mathrm{dia}^{-1}$, na queda; $256,81^{\circ} \mathrm{C} \mathrm{dia}{ }^{-1}$, na formação; e $236,96{ }^{\circ} \mathrm{C} \mathrm{dia}^{-1}$, na maturação.

3-A duração do ciclo fenológico da mangueira variou entre 110 e 112 dias, para todo o ciclo produtivo, sendo a média de 26 dias para floração; a variação entre 32 e 33 dias para queda; a média de 27 dias para formação e variação entre 25 e 26 dias para maturação.

\section{AGRADECIMENTOS}

Os autores agradecem ao Conselho Nacional de Desenvolvimento Científico e Tecnológico (CNPq), pela concessão da bolsa de estudos ao primeiro autor (Edital $\mathrm{n}^{\circ} 70 / 2009$, processo 557647/2010-0), e pelos demais recursos financeiros. À Universidade Federal Rural da Amazônia (UFRA), pela concessão da área de estudo, e ao Programa de Pós-Graduação em Agronomia da UFRA, ao Programa de Grande Escala da Biosfera-Atmosfera na Amazônia (LBA) e INCT, pelo auxílio logístico.

\section{REFERÊNCIAS}

ANDRADE, R.G; SEDIYAMA, G.C.; ZOLNIER, S.; COSTA, L.C. Avaliação de métodos para estimativa da temperatura-base para as culturas de milho (Zea mays L.) e feijão (Phaseolus vulgaris L.). Revista Brasileira de Agrometeorologia, Santa Maria, v.13, n.3, p.316-325, 2005.

BALLY, I.S.E.; HARRIS, M.; WHILEY, A.W. Effect of water on flowering and yield of Kensington Pride mango (Mangifera indica L.). Acta Horticulturae, Wageningen, v.509, p.277-282, 2000.
BARROS, M.P. de; ZANETTI, V.B.; FRAGA, C.I. de M.; NINCE, P.C. do C.; CAMPELO JÚNIOR, J.H.; LOBO, F. de A. Unidades fototérmicas e temperatura-base inferior de frutos de mangueira Alfa, na baixada cuiabana. Revista Brasileira de Fruticultura, Jaboticabal, v.32, n.2, p.479-485, 2010.

BERILLI, S. da S.; OLIVEIRA, J.G. de; MARINHO, A.B.; LYRA, G.B.; SOUSA, E.F. de; VIANA, A.P.; BERNARDO, S.; PEREIRA, M.G. Avaliação da taxa de crescimento de frutos de mamão (Carica papaya $\mathrm{L}$.) em função das épocas do ano e graus-dias acumulados. Revista Brasileira de Fruticultura, Jaboticabal, v.29, n.1, p.11-14, 2007.

BURONDKAR, M.M.; BHINGARDE, R.T.; KORE, V.N.; POWAR, A.G. Estimation of heat units as maturity indices for different mango varieties in Konkan region of Maharshtra. Acta Horticulturae, Wageningen, n.509, p.297-299, 2000.

CAMARGO, M.B.P.; BRUNINI, O.; MIRANDA, M.A.C. Temperatura-base para cálculo dos grausdia para cultivares de soja em São Paulo. Pesquisa Agropecuária Brasileira, Brasília, v.22, n.2, p.115121, 1987.

CAMPBELL, R.J.; CAMPBELL, C.W. The Tommy Atkins mango. Journal of the American Pomological Society, Nova Zelândia, v.60, p.5557, 2006.

CARVALHO, S.L.C. de; NEVES, C.S.V.J.; BÜRKLE, R.; MARUR, C.J. Épocas de indução floral e soma térmica do período do florescimento à colheita de abacaxi 'Smooth Cayenne'. Revista Brasileira de Fruticultura, Jaboticabal, v.27, n.3, p.430-433, 2005.

CHAVARRIA, G.; SANTOS, H.P. dos; MANDELLI, F.; MARODIN, G.A.B.; BERGAMASHI, H.; CARDOSO, L.S. Caracterização fenológica e requerimento térmico da cultivar Moscato Giallo sob cobertura plástica. Revista Brasileira de Fruticultura, Jaboticabal, v.31, n.1, p.119-126, 2009.

ISSARAKRAISILA, M.; CONSIDINE, J.A. Effects of temperature on pollen viability in mango cv. 'Kensington'. Annals of Botany, Exeter, v.73, p.231-240, 1994. 
LUCAS, D.D.P.; STRECK, N.A.; BORTOLUZZI, M.P.; TRENTIN, R.; MALDANER, I.C. Temperatura base para emissão de nós e plastocrono de plantas de melancia. Revista Ciência Agronômica, Fortaleza, v.43, n.2, p.288-292, 2012.

LUCENA, E.M.P. de; ASSIS, J.S. de; ALVES, R.E.; SILVA, V.C.M. da; ENÉAS FILHO, J. Alterações físicas e químicas durante o desenvolvimento de mangas ‘Tommy Atkins' no Vale do São Francisco, Petrolina-PE. Revista Brasileira de Fruticultura, Jaboticabal, v.29, n.1, p.096-101, 2007.

MOSQUEDA-VÁZQUEZ, R.; ROSA, F.; IRETAOJEDA, A. Degree-days and base temperatures required for inflorescence and fruit development in mango 'Manila'. Acta Horticulturae, Wageningen, n.341, p.232-239, 1993.

NUÑEZ-ELISEA，R.; DAVENPORT，T.L.; CALDEIRA, M.L. Control of bud morphogenesis in mango (Mangifera indica L.) by girdling defoliation and temperature modification. Journal of Horticultural Science, Ashford, v.71, p.25-40, 1996.

PRELA, A.; RIBEIRO, A.M. de A. Determinação de graus-dia acumulados e sua aplicação no planejamento do cultivo de feijão-vagem (Phaseolus vulgaris L.) para Londrina-PR. Revista Brasileira de Agrometeorologia, Santa Maria, v.10, n.1, p.8388, 2002.

RAMÍREZ, F.; DAVENPORT, T.L. Mango (Mangifera indica L.) flowering physiology. Scientia Horticulturae, Kidlington, v.126, p.65-72, 2010.

RANA, G.; KATERJI, N. Measurement and estimation of actual evapotranspiration in the field under Mediterranean climate: a review. European Journal of Agronomy, Kidlington, v.13, p.125153,2000 .
RIBEIRO, R.V.; MACHADO, E.C.; OLIVEIRA, R.F. de. Temperature response of photosynthesis and its interaction with light intensity in sweet orange leaf discs under non-photorespiratory condition. Ciência e Agrotecnologia, Lavras, v.30, n.4, p.670 $678,2006$.

SOARES, J.M.; COSTA, F.F.; SANTOS, C.R. Manejo de irrigação em frutíferas. In: FARIA, M.S.; SILVA, É.L.; VILELA, L.A.A.; SILVA, A.M. Manejo de irrigação. Poços de Caldas: DE/UFLA, 1998. p.281-308.

SOUZA, A.P. de; SILVA, A.C. da; LEONEL, S.; ESCOBEDO, J.F. Temperaturas basais e soma térmica para a figueira podada em diferentes épocas. Revista Brasileira de Fruticultura, Jaboticabal, v.31, n.2, p.314-322, 2009.

STRECK, N.A. A generalized nonlinear air temperature response function for node appearance rate in muskmelon (Cucumis melo L.). Revista Brasileira de Agrometeorologia, Santa Maria, v.10, n.1, p.105-111, 2002.

TRENTIN, R.; SCHREIBER, F.; STRECK, N.A.; BURIOL, G.A. Soma térmica de subperíodos do desenvolvimento da planta de melancia. Ciência Rural, Santa Maria, v.38, n.9, p.2464-2470, 2008.

YANG, S.; LOGAN, J.; COFFEY, D.L. Mathematical formulae for calculating the base temperature for growing degree days. Agricultural and Forest Meteorology, Amsterdam, v.74, p.61-74, 1995. 